Revista Interdisciplinaria de Humanidades, Educación, Ciencia y Tecnología

Año VI. Vol. VI. N¹. Edición Especial. 2019

Hecho el depósito de ley: pp201602FA4721

ISSN-L: 2542-3029; ISSN: 2610-802X

Universidad Nacional Experimental Francisco de Miranda (UNEFM). Santa Ana de Coro. Venezuela

Rudyard Antonio Arteaga Solorzano; Freddy Alain Mendoza Rivadeneira; Roy Leonardo Barre Zambrano;

Plinio Abelardo Vargas Zambrano

DOI 10.35381/cm.v5i1.261

\title{
Determinación del tipo de cuajado en el queso fresco artesanal en Manabí Ecuador
}

\section{Determination of the type of curd in the artisan fresh cheese in Manabí Ecuador}

\author{
Rudyard Antonio Arteaga Solorzano \\ rarteaga@utm.edu.ec \\ Universidad técnica de Manabí, Portoviejo \\ Ecuador \\ https://orcid.org/0000-0001-5499-7334 \\ Freddy Alain Mendoza Rivadeneira \\ famendoza@utm.edu.ec \\ Universidad técnica de Manabí, Portoviejo \\ Ecuador \\ https://orcid.org/0000-0003-1457-688X \\ Roy Leonardo Barre Zambrano \\ rolebaz@hotmail.com \\ Universidad Laica Eloy Alfaro de Manabí, Manta \\ Ecuador \\ https://orcid.org/0000-0002-4849-3532 \\ Plinio Abelardo Vargas Zambrano \\ pavargas@utm.edu.ec \\ Universidad técnica de Manabí, Portoviejo \\ Ecuador \\ https://orcid.org/0000-0002-2152-7317
}

Recibido: 1 de septiembre de 2019

Aprobado: 30 de Septiembre de 2019

\section{RESUMEN}

El presente estudio aborda los métodos de cuajado utilizados en la provincia de Manabí, específicamente en los cantones de Flavio Alfaro, Chone, Pedernales y el Carmen, se tomaron 262 muestras de la población total de productores de la zona con el objetivo de 
determinar las técnicas de cuajado más usadas, la apertura de los productores a cambiar de método de cuajado. en Flavio Alfaro se utiliza principalmente cuajo liofilizado agregado después de la recepción, Chone usa en mayoría cuajo en pastilla agregado después del tamizado, el Carmen cuajo líquido agregado después de la recepción y Pedernales cuajo en pastilla agregado después de la recepción, solamente el Carmen se encuentra dispuesto a cambiar de método de cuajado, actualmente solo el $15 \%$ de los productores utilizan pasteurización de leche para la elaboración de queso fresco, la falta de pasteurización, se recomienda generar centros de acopio lechero.

Descriptores: Nutrición animal, ganado vacuno, producto lácteo, industria lechera.

\begin{abstract}
The present study addresses the curdling methods used in the province of Manabí, specifically in the cantons of Flavio Alfaro, Chone, Pedernales and El Carmen, 262 samples were taken from the total population of producers in the area in order to determine the techniques of curd more used, the opening of the producers to change of method of curdling. Flavio Alfaro mainly uses lyophilized rennet added after reception, Chone mostly uses rennet in added tablet after sieving, Carmen rennet liquid added after reception and Pedernales rennet in added tablet after reception, only Carmen se is willing to change curdling method, currently only $15 \%$ of the producers use milk pasteurization to make fresh cheese, the lack of pasteurization, it is recommended to generate milk collection centers.
\end{abstract}

Descriptors: Animal nutrition, cattle, dairy products, dairy industry.

\title{
INTRODUCCIÓN
}

El queso es un alimento de gran importancia en el mundo, su principal materia prima es la leche, la cual es obtenida de mamíferos mayores como las vacas, las búfalas y las cabras. El queso es reconocido por su alto valor nutricional, consta de entre $20-25 \%$ de proteína altamente digerible (Galván, 2005). El queso de vaca es el de mayor importancia económica en el Ecuador, debido a las cantidades producidas, en el 2011 se produjeron aproximadamente 97500 toneladas métricas de queso a partir de leche entera de vaca, tomando en cuenta la producción de 91000 toneladas del 2010 se registra un aumento del $7.1 \%$ en la producción de este producto (FAO, 2012). 


\section{CIENCIAMATRIA}

Revista Interdisciplinaria de Humanidades, Educación, Ciencia y Tecnología

Año VI. Vol. VI. N¹. Edición Especial. 2019

Hecho el depósito de ley: pp201602FA4721

ISSN-L: 2542-3029; ISSN: 2610-802X

Universidad Nacional Experimental Francisco de Miranda (UNEFM). Santa Ana de Coro. Venezuela

Rudyard Antonio Arteaga Solorzano; Freddy Alain Mendoza Rivadeneira; Roy Leonardo Barre Zambrano;

Plinio Abelardo Vargas Zambrano

El cuajado del queso es una operación unitaria que realizada sin excepción en la elaboración del queso, en esta operación se agrega el cuajo una sustancia que contiene quimosina una enzima de la familia de las hidrolasas cuya principal función es la precipitación de la caseína para la formación de la cuajada, producto intermedio que posteriormente se procesa en queso (Bylund, 1995). El presente estudio busca cuantificar por medio de encuestas y medición directa los procedimientos de cuajado que se usan en la provincia de Manabí para la elaboración del queso fresco artesanal, así como dar una idea de la producción realizada en los sectores productivos de Manabí.

Este estudio brindará una perspectiva de los métodos de elaboración del queso artesanal en la zona de Manabí y brindará ideas generales de las opciones disponibles para la mejora continua de la matriz productiva. El gobierno del Ecuador en el año 2013 por medio del "Plan Nacional del Buen Vivir" en base al cual se realiza la planificación estratégica de acción gubernamental para el crecimiento del país y realización de obras clave determino: la necesidad de impulsar el desarrollo del pequeño y mediano productor para un crecimiento sostenido de la seguridad alimentaria del país, la importancia de la tecnificación mecánica y la incorporación de conocimientos al desarrollo de la matriz productiva y la inclusión de la población en las microempresas productoras para generación de empleos y rentas; siendo esto de gran impacto en la industria quesera, que presenta un consumo del $25 \%$ de la leche que se produce en el Ecuador (Correa, et al., 2013).

En la actualidad existen programas del MAGAP (Ministerio de Agricultura, Ganadería, Acuacultura y Pesca) que son dedicados al aumento de la matriz productiva, tales como PRONERI (Programa Nacional de Negocios Rurales Inclusivos) y el programa de ganadería sostenible, los cuales se dedican a que los productores medianos y grandes, puedan aumentar su producción y tecnificarla a través de redes de acopio de leche y la capacitación de productores, más estos programas en desarrollo no están disponibles aun para todos los ganaderos.

El queso fresco se realiza artesanalmente con el filtrado de la leche por medio de mantas 


\section{CIENCIAMATRIA}

Revista Interdisciplinaria de Humanidades, Educación, Ciencia y Tecnología

Año VI. Vol. VI. N¹. Edición Especial. 2019

Hecho el depósito de ley: pp201602FA4721

ISSN-L: 2542-3029; ISSN: 2610-802X

Universidad Nacional Experimental Francisco de Miranda (UNEFM). Santa Ana de Coro. Venezuela

Rudyard Antonio Arteaga Solorzano; Freddy Alain Mendoza Rivadeneira; Roy Leonardo Barre Zambrano;

Plinio Abelardo Vargas Zambrano

o telas, la cual posteriormente se le agrega cuajo y se reposa alrededor de 45 minutos, la cuajada resultante de la precipitación de la caseína en esta operación se rompe desigualmente por medio de acción mecánica, normalmente mediante el uso de las manos, posteriormente se filtra y escurre la cuajada, se le agrega la sal, se prensa por al menos 24 horas y se empaca (García, et al., 1987).

Según Borjas (1998) existen cuatro factores de interés en el adecuado cuajado del queso: la cantidad de quimosina que se agrega a la leche para la precipitación de la caseína, la temperatura a la que se genera la reacción, que regularmente se aproxima a los $30^{\circ} \mathrm{C}$, la acidez de la leche que puede ser dada por medio de cultivos lácticos a escala industrial y por medio de las bacterias presentes en el proceso en el caso de los procesos industriales, más en ambos casos la acides mínima recomendada es de $0.02 \%$ de ATECAL y la concentración de sales de calcio, las cuales solo disminuyen debido a procesos de pasteurización que normalmente no están presentes en la elaboración.

Según (Aray, Intriago, \& Mendoza, 2013) en Manabí se estima una población de aproximadamente 260000 vacas lecheras, que producen aproximadamente $1000000 \mathrm{~L}$ de leche/día, esto representa el $20 \%$ de la producción de lácteos del Ecuador solo en esta Zona (FAO, 2012).

En la actualidad no se conocen exactamente la cantidad de leche destinada a la elaboración de queso en la provincia de Manabí, el MAGAP determinó que en el año 2012 para la zona 4 que comprende la provincias de Manabí y Santo Domingo de los Tachillas se producen $663000 \mathrm{~L}$ de leche diario, de esta cantidad se procesa para elaborar queso artesanal una parte importante, según datos de la AGSO (Asociación de ganaderos de la sierra y el oriente) el $11 \%$ de la leche que se produce en la sierra y el oriente del Ecuador se utiliza para la elaboración de queso artesanal. Utilizando esta información como base de cálculo para el área de Manabí se podría estimar 72930 L de leche destinada a la elaboración de queso artesanal en esta área. 


\section{METODOLOGÍA}

La investigación fue de tipo descriptiva desde la perspectiva de Hernández, Fernández y Baptista (2014), se realizó en la zona norte de la provincia de Manabí-Ecuador por ser la mayor productora de queso fresco artesanal de la costa ecuatoriana, dentro de la misma Ios cantones: Chone, El Carmen, Flavio Alfaro y Pedernales representan el 90\% de esta producción de queso en la provincia. Para el presente estudio se consideraron los productores registrados en el proyecto de la CONEFA (Comisión Nacional de Erradicación de Fiebre Aftosa) 2016 de los cantones Chone, Flavio Alfaro, Pedernales y El Carmen) y, para lo cual se utilizó la siguiente ecuación de muestreo que cita Morales (2011), para población finita indicada para universo de entre 100-5000.

$$
n=\frac{N}{1+\frac{e^{2}(N-1)}{Z^{2} p q}}
$$

Donde:

$\mathrm{n}=$ tamaño de muestra a estudiar

$\mathrm{N}=$ Población

$\mathrm{e}=$ Error muestral

$Z$ = nivel de confianza

$\mathrm{p} \mathrm{q}=$ Varianza de la población

En el detalle del cuadro que se desarrolló a continuación resumen los datos y la muestra estimada que formó parte del estudio: 


\section{Cuadro 1}

Determinación de muestra para observación in situ

\begin{tabular}{cccccc} 
Cantón & Población (N) & $\mathbf{p ~ q}$ & Error (e) & $\mathbf{Z}$ & Muestra \\
\hline Chone & 236 & 0,5 & 0,5 & 1,96 & 147 \\
Flavio Alfaro & 283 & 0,5 & 0,5 & 1,96 & 163 \\
Pedernales & 187 & 0,5 & 0,5 & 1,96 & 126 \\
El Carmen & 185 & 0,5 & 0,5 & 1,96 & 126 \\
\hline
\end{tabular}

\section{Variables evaluadas}

\begin{tabular}{|c|c|c|c|}
\hline $\begin{array}{c}\text { Conceptualización } \\
\text { de las variables }\end{array}$ & $\begin{array}{c}\text { Dimensión o } \\
\text { Categoría }\end{array}$ & Indicador & Instrumentos \\
\hline $\begin{array}{c}\text { TCU: } \\
\text { Tipo de Cuajo } \\
\text { Usado }\end{array}$ & $\begin{array}{c}\text { Variable } \\
\text { Dependiente }\end{array}$ & $\begin{array}{ll}\text { - } & \text { Fresco } \\
\text { - } & \text { Pastilla } \\
\text { - } & \text { Liofilizado } \\
\text { - } & \text { Cuajo } \\
& \text { Líquido }\end{array}$ & $\begin{array}{l}\text { Encuesta } \\
\text { Fotos }\end{array}$ \\
\hline $\begin{array}{c}\text { MCL: } \\
\text { Momento del } \\
\text { Cuajado de la } \\
\text { Leche }\end{array}$ & $\begin{array}{c}\text { Variable } \\
\text { Dependiente }\end{array}$ & $\begin{array}{ll}\text { - } & \text { Recepción } \\
\text { - } & \text { Después del } \\
& \text { pasteurizado } \\
\text { - } & \text { Después del } \\
\text { tamizado }\end{array}$ & $\begin{array}{l}\text { Encuesta } \\
\text { Fotos }\end{array}$ \\
\hline $\begin{array}{c}\text { DCT: } \\
\text { Disposición de } \\
\text { Cambiar la } \\
\text { Técnica }\end{array}$ & $\begin{array}{c}\text { Variable } \\
\text { Dependiente }\end{array}$ & $\begin{array}{ll}- & S i \\
- & \text { No }\end{array}$ & $\begin{array}{l}\text { Encuesta } \\
\text { Fotos }\end{array}$ \\
\hline $\begin{array}{l}\text { LE: } \\
\text { Lugar de estudio }\end{array}$ & $\begin{array}{c}\text { Variable } \\
\text { Independiente }\end{array}$ & $\begin{array}{ll}\text { - } & \text { Chone } \\
\text { - } & \text { El Carmen } \\
\text { - } & \text { Flavio Alfaro } \\
\text { - } & \text { Pedernales }\end{array}$ & Mapas \\
\hline
\end{tabular}




\section{RESULTADOS Y DISCUSIÓN}

\section{Gráfico 1}

Regiones según el tipo de cuajo usado expresado en fincas.

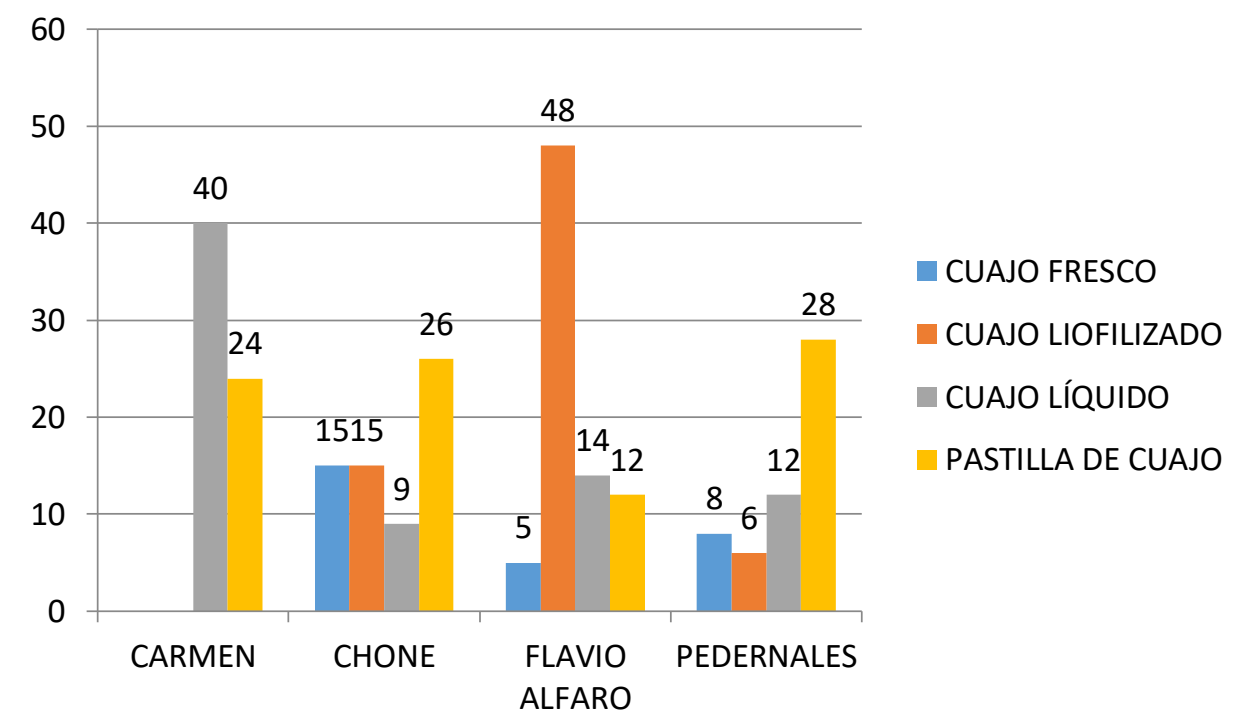

Como se puede observar en el gráfico 1 en El Carmen tiene 40 fincas que utilizan cuajo líquido, es decir el 62.5\% de la población prefiere usar cuajo líquido, siendo el único lugar de estudio donde la mayor parte de la población utiliza cuajo líquido.

El Chone la preferencia más común es el cuajo en pastilla con el $40 \%$ de uso entre la población, superior al $23 \%$ de preferencia de cuajo fresco y cuajo liofilizado las cuales o el $14 \%$ de uso que tiene el cuajo líquido.

Flavio Alfaro presento una preferencia del $61 \%$ con respecto al 18,15 y $6 \%$ que hubo para el cuajo líquido, la pastilla de cuajo y el cuajo fresco respectivamente; mientras que para Pedernales existió una preferencia mayor para la pastilla de cuajo donde un $52 \%$ de la población la eligió sobre las otras alternativas, que en ningún caso sobrepasan al 30 $\%$ de aceptación.

La preferencia de un tipo de coagulante sobre otros de forma tan marcada en cada uno de los lugares de estudio se explica con 2 factores: La disponibilidad geográfica de cada uno de los insumos y la asimilación de conceptos y adaptación a los mismos que se 
realiza como actividad de carácter social, haciendo que comunidades vecinas adapten métodos y costumbres de trabajo parecidas unas entre otras.

La preferencia en Manabí es de usar cuajo en pastilla, un 34\% de los productores lo usan, $29 \%$ de los productores usan cuajo líquido, $26 \%$ utilizan cuajo liofilizado y el $11 \%$ usan cuajo fresco.

\section{Gráfico 2}

Regiones según el momento de adición del cuajo

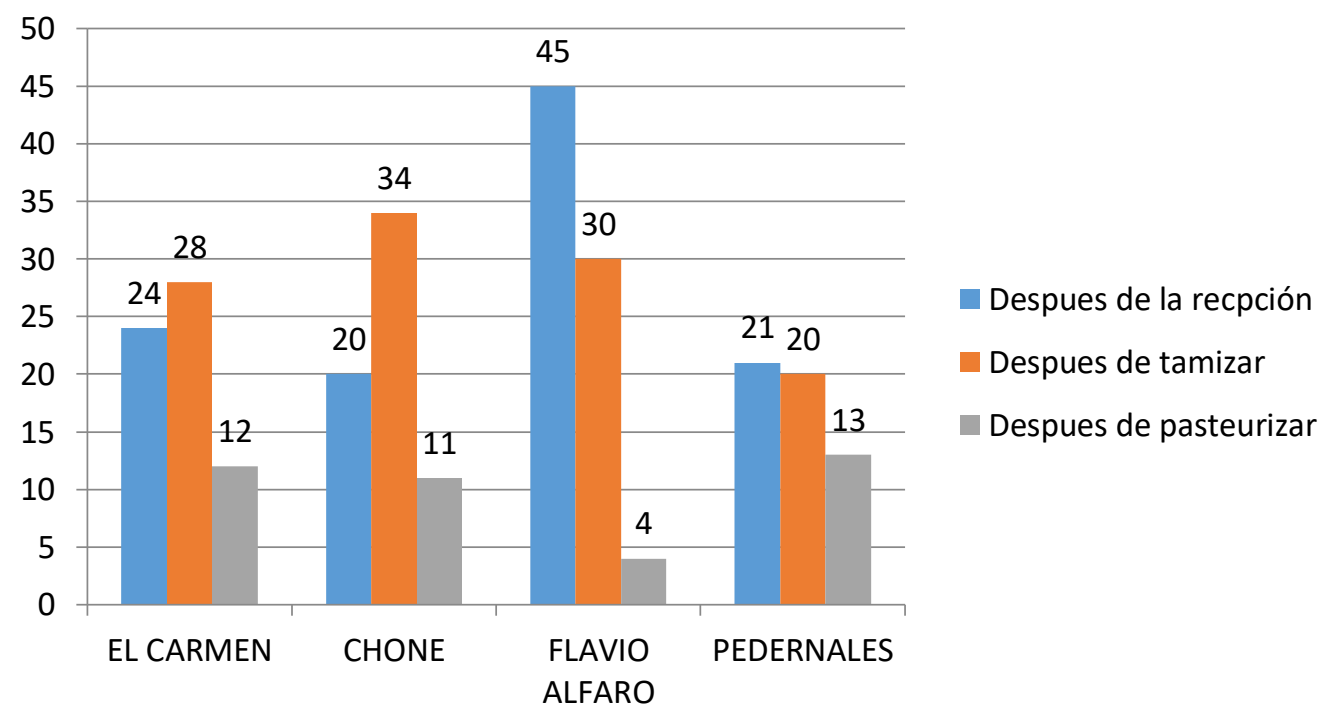

Como en el gráfico 2 no existe preferencia por agregar el cuajo después del proceso de pasteurizado, que es el método más inocuo según Borjas (1998), esto se debe principalmente a que los productores carecen de la tecnología para pasteurizar la leche, el $100 \%$ de los productores que realizan el proceso de pasteurización agregan el cuajo después del mismo.

En Flavio Alfaro y Pedernales existe una preferencia para el método de agregar el cuajo después de la recepción del 56 y 38\%, mientras, que en el Carmen y Chone se agrega el cuajo después del filtrado en un 44 y $52 \%$ respectivamente. 


\section{CIENCIAMATRIA}

Revista Interdisciplinaria de Humanidades, Educación, Ciencia y Tecnología

Año VI. Vol. VI. N¹. Edición Especial. 2019

Hecho el depósito de ley: pp201602FA4721

ISSN-L: 2542-3029; ISSN: 2610-802X

Universidad Nacional Experimental Francisco de Miranda (UNEFM). Santa Ana de Coro. Venezuela

Rudyard Antonio Arteaga Solorzano; Freddy Alain Mendoza Rivadeneira; Roy Leonardo Barre Zambrano;

Plinio Abelardo Vargas Zambrano

Al no realizar un proceso de pasteurización de la leche el queso resultante tiene mayor carga bacteriana y un sabor característico, normalmente este proceso no se realiza en las producciones de queso en Manabí, solamente el 15\% de las fincas producen queso utilizando procesos de pasteurización.

\section{Gráfico 3}

Disposición a cambiar método de cuajado

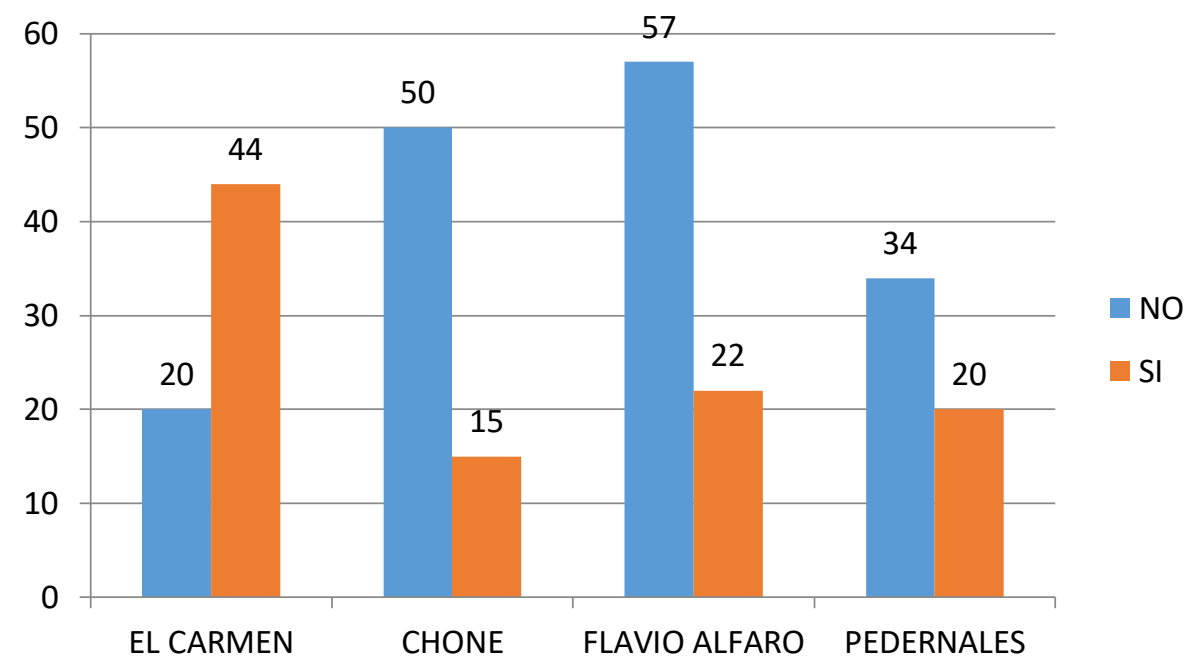

Como se observa en el cuadro 3 solamente en El Carmen existe una mayoría de productores dispuestos a cambiar de método de cuajado en la elaboración de queso, es decir que el $69 \%$ de los productores están dispuestos a cambiar su método de cuajado, ya sea por hacer este más eficiente, aumentar la producción o porque están inconformes con el método que usan actualmente, desde una perspectiva global, el $61 \%$ de los productores no quieren cambiar de método usado debido a que actualmente están conformes con el que aplican. 


\section{Gráfico 4}

Opinión de productores respecto a su método de cuajado

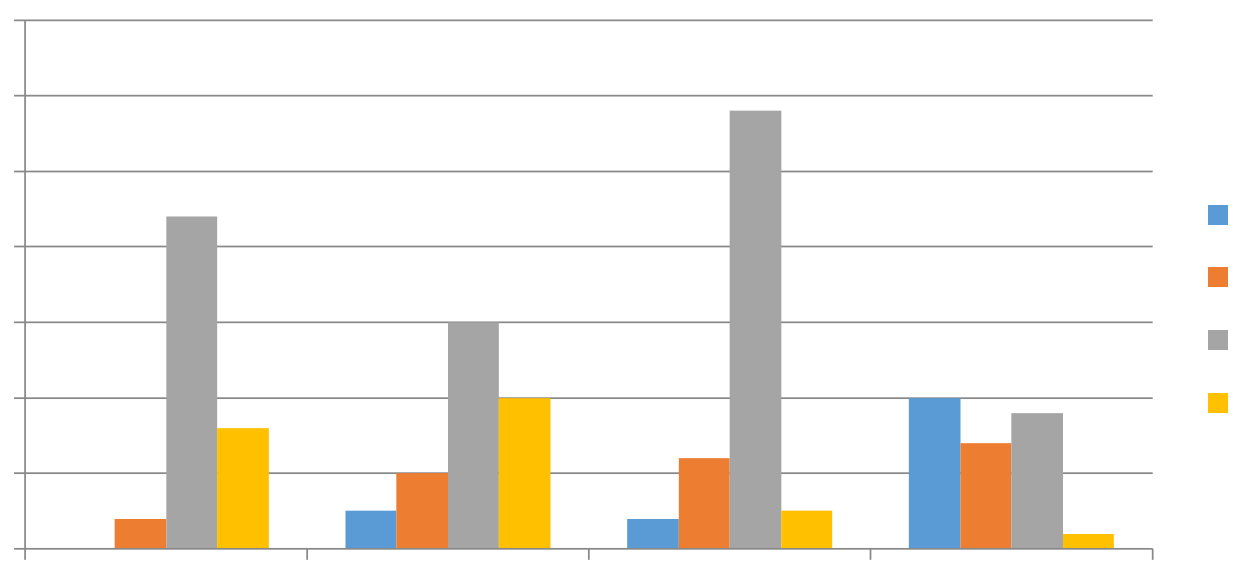

Como se observa en el gráfico 4, la percepción del 57\% de los productores es que el método que se encuentran usando actualmente es el mejor, lo cual concuerda con la información expresada en el gráfico 3 que expresa la renuencia general de los productores a cambiar método de elaboración de queso.

El método de cuajado en la producción de queso actualmente se encuentra en proceso de estancación debido a la falta de deseo de cambio entre los productores, con la única excepción del Carmen donde la mayoría de productores encuestados está dispuesto a cambiar de técnica, en un $68 \%$ de los casos para mejorar la producción en rendimiento y en $32 \%$ para mejorar la calidad e inocuidad de la misma. 


\section{CONCLUSIONES}

En Manabí se usa principalmente el cuajo en pastilla en un 34\% de las producciones, el cual se agrega a leche no pasteurizada en un $85 \%$ de los casos.

Solo en El Carmen los productores se encuentran abiertos a cambiar su método de elaboración de queso, en el $69 \%$ de los casos desean métodos que mejorar el rendimiento de la producción.

El $15 \%$ de la leche que se produce en la Zona Norte de Manabí es pasteurizada.

\section{REFERENCIAS CONSULTADAS}

1. Aray, M., Intriago, M. \& Mendoza, H. (2013). Producción y comercialización de queso mozarella en la ciudad de Chone, provincia de Manabí. Tesis para obtar al título de Ingeniero Comercial, Departamento de Economía, Univerciada Técnica de Manabí, Manabí, Ecuador.

2. Borjas, E. (1998). Tecnificación de los procesos de manufactura y caracterización de quesos artesanales centroamericanos para exportación. Tesis para optar al título de Ingeniero Agroindustrial, Departamento de Agroindustria Alimentaria, Escuela Agrícola Panamericana el Zamorano, Francisco Morazan, Honduras.

3. Carmona, M. Rubio, C. \& Lemus, C. (2002). Estadística Aplicada a la Investigación. Nayarit: México

4. Correa, R., Glas, J., Muñoz, P., Vaca, C., Long, G., Arellano, H., Rivera, P., Poveda, R., Espinosa, R., Ramírez, R., Sánchez, M., Martínez, J., Chilán, C., Torre, P., Maji, R., Quiñónez, Y., Rodríguez, K. \& Larrea, A. (2013). Plan nacional del buen vivir. Quíto: Gobierno de la República del Ecuador.

5. Bylund, G. (1995). Dairy processing handbook. Lund: Tetrapack.

6. FAO. (2012). Datos de producción de queso a partir de leche entera de vaca. Italia: FAO. 


\section{CIENCIAMATRIA}

Revista Interdisciplinaria de Humanidades, Educación, Ciencia y Tecnología

Año VI. Vol. VI. N¹. Edición Especial. 2019

Hecho el depósito de ley: pp201602FA4721

ISSN-L: 2542-3029; ISSN: 2610-802X

Universidad Nacional Experimental Francisco de Miranda (UNEFM). Santa Ana de Coro. Venezuela

Rudyard Antonio Arteaga Solorzano; Freddy Alain Mendoza Rivadeneira; Roy Leonardo Barre Zambrano;

Plinio Abelardo Vargas Zambrano

7. Galván, M. (2005). Proceso básico de la leche y el queso. Revista digital universitaria UNAM , 167-184.

8. García, O., Ochoa, I., Novoa, C., Baylon, C., Granados, F., Duque, O., \& Murcia, R. (1987). Derribados Lacteos: Procesamiento de quesos blancos. Bogota: Centro Agropecuario de la Sabana.

9. Hernández, R., Fernández, C., \& Baptista, P. (2014). Metodología de la investigación. México, Mc Graw Hill Hispanoamericana. Hill Internacional.

10. MAGAP. (2012). Sistema de Información Nacional de Agricultura, Ganadería Acuacultura y Pesca. Retrieved from Reporte de Resultados del Censo Provincial Completo en excel (Todas las Provincias): http://sinagap.agricultura.gob.ec/resultados-provinciales

\section{REFERENCES CONSULTED}

1. Aray, M., Intriago, M. \& Mendoza, H. (2013). Production and marketing of mozzarella cheese in the city of Chone, province of Manabí. Thesis to obtain the title of Commercial Engineer, Department of Economics, Technical University of Manabí, Manabí, Ecuador.

2. Borjas, E. (1998). Technification of the manufacturing processes and characterization of Central American artisanal cheeses for export. Thesis to qualify for the degree of Agroindustrial Engineer, Department of Food Agribusiness, Pan American Agricultural School El Zamorano, Francisco Morazan, Honduras.

3. Carmona, M. Rubio, C. \& Lemus, C. (2002). Applied Statistics to Research. Nayarit: Mexico

4. Correa, R., Glas, J., Muñoz, P., Vaca, C., Long, G., Arellano, H., Rivera, P., Poveda, R., Espinosa, R., Ramírez, R., Sánchez, M., Martínez, J., Chilán, C., Torre, P., Maji, R., Quiñónez, Y., Rodríguez, K. \& Larrea, A. (2013). National plan of good living. Chemical: Government of the Republic of Ecuador.

5. Bylund, G. (1995). Dairy processing handbook. Lund: Tetrapack.

6. FAO. (2012). Cheese production data from whole cow's milk. Italy: FAO. 
Revista Interdisciplinaria de Humanidades, Educación, Ciencia y Tecnología

Año VI. Vol. VI. N¹. Edición Especial. 2019

Hecho el depósito de ley: pp201602FA4721

ISSN-L: 2542-3029; ISSN: 2610-802X

Universidad Nacional Experimental Francisco de Miranda (UNEFM). Santa Ana de Coro. Venezuela

Rudyard Antonio Arteaga Solorzano; Freddy Alain Mendoza Rivadeneira; Roy Leonardo Barre Zambrano;

Plinio Abelardo Vargas Zambrano

7. Galván, M. (2005). Basic process of milk and cheese. University digital magazine UNAM, 167-184.

8. García, O., Ochoa, I., Novoa, C., Baylon, C., Granados, F., Duque, O., \& Murcia, R. (1987). Demolished Dairy Products: White cheese processing. Bogota: Agricultural Center of La Sabana.

9. Hernández, R., Fernández, C., \& Baptista, P. (2014). Investigation methodology. Mexico, Mc Graw Hill Hispanic American. Hill International

10. MAGAP (2012). National Information System of Agriculture, Livestock Aquaculture and Fisheries. Retrieved from Report of Results of the Complete Provincial Census in excel (All Provinces): http://sinagap.agricultura.gob.ec/resultados-provinciales

(C)2019 por los autores. Este artículo es de acceso abierto y distribuido según los términos y condiciones de la licencia Creative Commons Atribución-NoComercial-Compartirlgual 4.0 Internacional (CC BY-NC-SA 4.0) (https://creativecommons.org/licenses/by-nc-sa/4.0/). 